\title{
Therapeutic Presence - Virtual Illusions for Neurorehabilitation
}

\author{
Thomas Schüler \\ Institute for Cognitive Science, University of Osnabrueck \\ thschuel@uni-osnabrueck.de \\ http://www.thomasschueler.de
}

\begin{abstract}
The poster presents a project in which a computer game for neuro-rehabilitation is developed and its application explored. The development is based on the principles of mirror therapy and draws on recent findings in research on the concept of presence. Specifically, it is assumed that artistic virtual environments (VE) induce high presence experiences and thus enhance the treatment of neurologic diseases.
\end{abstract}

Keywords: virtual rehabilitation, neuro-rehabilitation, presence.

\section{Introduction}

Visual illusions may be used in neuro-rehabilitation to achieve therapeutic effects. This has been shown in 1995 by Ramachandran, Rogers-Ramachandran and Cobb who developed the "mirror therapy" based on these findings [1. In mirror therapy patients with unilateral neurologic diseases are treated by a visual illusion that establishes the impression of control over the affected side. A mirror is placed on the sagittal body axis in front of the patient such that the reflected image of the unaffected body side overlays the actual position of the affected body parts. The patient is then asked to perform movements with both sides while watching into the mirror. This way the coupling between intended and perceived actions gives the impression of actually performing these actions with the affected body parts, thus leading to therapeutic effects on different neurologic diseases [2].

In recent years, systems have been proposed that transfer the idea of mirror therapy to virtual reality. 3] and [4] developed systems for patients with upper limb disabilities. In both systems the patients control an avatar through their natural movements and perform tasks, e.g. hitting flying balls with the avatar's hands. When these systems are used for mirror therapy, the movements of the patients' unaffected limb are used to control both limbs of the avatar, thus displaying symmetrically mirrored movements. The tasks are chosen such that symmetrical movements are needed to perform successfully. This way the visual illusion of a moving humanoid limb at the patients' affected side is established in a VE. In pilot studies, patients suffering from phantom limb pain and hemiparesis following a stroke have been successfully treated with these systems (ib.).

An explanation for the effectiveness of the mirror therapy is "the brain's predilection for prioritizing visual feedback over somatosensory/proprioceptive 
feedback concerning limb position" 5]. The visual illusion of a moving limb triggers those motor cortex regions of the observer that usually control the corresponding limb [6]. The degree to which the illusions are experienced as real plays an important role in the effectiveness of the treatment 7. Using VEs for the therapy, the visual illusions can be modified in various ways to enhance the effect. This may be inspired by the area of computer game design. In research on VEs the term "presence" refers to the subjective experience of an agent that perceives and reacts to sensorial stimuli of a VE in a way similar to real life [8].

It is hypothesized here that the presence experience is central to the effectiveness of mirror therapy. The poster presents a project in which a computer game for neuro-rehabilitation is developed following this hypothesis. The game builds on the principles of mirror therapy and will be used with amputees to alleviate phantom limb pain. Specifically, it is assumed that artistic VEs induce high presence experiences and thus enhance the treatment of neurologic diseases.

\section{Presence and Action}

In the framework proposed by [9] and [10, presence is related to "the (prereflexive) perception of successfully transforming intentions in actions" [9]. For a strong experience of presence in relation to a VE, a correspondence between actions and intentions is more important than visual realism. Even a perceptually poor VE may constitute a strong experience of presence if it successfully supports an agent in carrying out his/her intended actions.

The framework is grounded in the view that all human behaviour in any environment is hierarchically composed of low-level operations that form actions and high-level activities [1]. These layers are all driven by some form of intentions and the successful (hypothetical) execution of the intentions by the agent generates presence as a sense of being-in-the-world 9. Low-level operations (e.g. motor operations) and their intentions are usually highly automated and unconscious. However, if an intended operation fails to produce the expected result, higher-level cognitive processing is needed to reflect and alter the plan. This puts the agent in a more internal cognitive state and is experienced as a break-down in presence.

According to this framework, a patient with phantom limb pain experiences low presence regarding the phantom, when he/she is not able to enact his/her intentions with that limb. However, the visual illusion described above may provide the impression of successful action for the patient. It is important to mention that an agent evaluates the execution of behaviour mainly through monitoring its effect on an external world. Recent studies have demonstrated that an agent can in fact feel physically present even in a tool, if this tool is fully controlled by him/her 9].

\section{Implications for Neuro-rehabilitation}

Following this understanding when designing a VE for neuro-rehabilitation, it is particularly important to support the execution of all behaviour that can 
possibly be intended by patients. This can, however, be a sheer impossible task, because of the infinite amount of behavioural intentions a patient can have. Although intentions become more predictable when a concrete task is given and when it is clear which operations are necessary to accomplish the task, especially low-level operations are highly subjective and individual when patients have to accomplish these task by controlling humanoid limbs in a realistic VE. Moreover, if real-world movement of the patients is used to interact with the $\mathrm{VE}$, the recording of this movement is limited by hardware needs. Patients will experience low presence and thus will have difficulties to believe in the illusion.

On the other hand, an artistic VE consisting of simple objects that are controlled by natural movement of patients will not lead to these problems. The artistic feedback can be generated based on the continuous flow of movement data in various ways. The behavioural possibilities within the VE need to be explored by the patients and their intentions are then based on this experience. The illusionary movement of the arm itself does not need to be displayed in order to feel that the observed behaviour was initiated by such a movement. Such artistic VE may still be based on mirror therapy principles and display bilateral behaviour that is controlled by unilateral movement. Using this kind of VE seems promising for the purposes of neuro-rehabilitation.

\section{Artistic Virtual Environment for Neuro-rehabilitation}

The assumptions presented above form the basis of a research project. It is investigated whether the use of an artistic VE, which provides a strong experience of presence, will be beneficial for the rehabilitation of neurologic diseases. A computer game for upper limb amputees with phantom limb pain is developed and therefore an artistic VE is designed that will be controlled according to mirror therapy principles. Figure 1 shows a an early prototype of the VE in application.

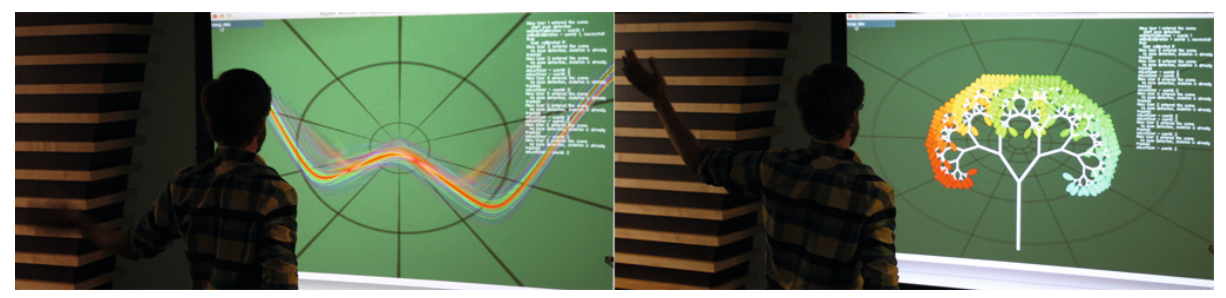

Fig. 1. A virtual environment for neuro-rehabilitation

The artistic feedback in the VE is generated using the movement of the patients' healthy limb and this movement is recorded using a motion capturing device. The body posture and gesture is recognized in the software and used as input data for generative design algorithms [12, 13. These algorithms transform structured input data into expressive artistic output. For the above described 
application algorithms are developed that generate the output continuously, such that every movement of the patient results in an immediate effect on the virtual environment. The algorithms are chosen to produce expressive bilateral output based on symmetrically mirrored unilateral input. While controlling this VE the experience of presence will be strong and the alleviation of phantom limb pain is assumed.

\section{Future Directions}

The VE will be evaluated in summer 2012 with a small sample of amputees suffering from phantom limb pain. During and after the sessions the patients will be asked to report on their presence experience and their level of pain.

The scientific focus of the project is on the application of the theoretical framework of presence presented above. The project will be completed by the end of 2012. The poster presents the theoretical framework, the developed game and presumably preliminary results of the sample study.

\section{References}

1. Ramachandran, V.S., Rogers-Ramachandran, D., Cobb, S.: Touching the phantom limb. Nature (1995)

2. Ramachandran, V.S., Altschuler, E.L.: The use of visual feedback, in particular mirror visual feedback, in restoring brain function. Brain (2009)

3. Murray, C.D., Pettifer, S., Howard, T., Patchick, E.L., Caillette, F., Kulkarni, J., Bamford, C.: The treatment of phantom limb pain using immersive virtual reality: Three case studies. Disability \& Rehabilitation (2007)

4. Eng, K., Siekierka, E., Pyk, P., Chevrier, E., Hauser, Y., Cameirao, M., Holper, L., Hägni, K., Zimmerli, L., Duff, A.: Interactive visuo-motor therapy system for stroke rehabilitation. Medical and Biological Engineering and Computing (2007)

5. Moseley, G.L., Gallace, A., Spence, C.: Is mirror therapy all it is cracked up to be? Current evidence and future directions. Pain (2008)

6. Iacoboni, M., Mazziotta, J.C.: Mirror neuron system: basic findings and clinical applications. Annals of Neurology (2007)

7. Rothgangel, A.: Spiegeltherapie - mehr als nur eine visuelle Illusion? pt_Zeitschrift für Physiotherapeuten (2008)

8. Slater, M.: A note on presence terminology. Presence Connect (2003)

9. Riva, G.: Is presence a technology issue? Some insights from cognitive sciences. Virtual Reality (2009)

10. Waterworth, J.A., Waterworth, E.L., Mantovani, F., Riva, G.: On Feeling (the) Present. Journal of Consciousness Studies (2010)

11. Kaptelinin, V., Nardi, B.A.: Acting with Technology: Activity Theory and Interaction Design. The MIT Press (2006)

12. McCormack, J., Dorin, A., Innocent, T.: Generative design: a paradigm for design research. In: Proceedings of Future Ground. Design Research Society (2004)

13. Bohnacker, H., Groß, B., Laub, J.: Generative Gestaltung. Verlag Hermann Schmidt (2009) 